\title{
Inverse pressure-induced Mott transition in $\mathrm{TiPO}_{4}$
}

\author{
H. Johan M. Jönsson, ${ }^{1}$ Marcus Ekholm, ${ }^{1,2}$ Maxim Bykov, ${ }^{3,4}$ Leonid Dubrovinsky, ${ }^{4}$ Sander van Smaalen, ${ }^{3}$ \\ and Igor A. Abrikosov ${ }^{1,5}$ \\ ${ }^{1}$ Theoretical Physics Division, Department of Physics, Chemistry and Biology (IFM), Linköping University, SE-581 83, Linköping, Sweden \\ ${ }^{2}$ Swedish e-Science Research Centre (SeRC), Linköping University, SE-581 83, Linköping, Sweden \\ ${ }^{3}$ Laboratory of Crystallography, University of Bayreuth, 95440 Bayreuth, Germany \\ ${ }^{4}$ Bayerisches Geoinstitut, University of Bayreuth, 95440 Bayreuth, Germany \\ ${ }^{5}$ Materials Modeling and Development Laboratory, National University of Science and Technology “MISIS,” Moscow 119049, Russia
}

(Received 27 March 2019; published 18 June 2019)

\begin{abstract}
$\mathrm{TiPO}_{4}$ shows interesting structural and magnetic properties as temperature and pressure are varied, such as a spin-Peierls phase transition and the development of incommensurate modulations of the lattice. Recently, highpressure experiments for $\mathrm{TiPO}_{4}$ reported two structural phases appearing at high pressures, the so-called phases IV and V [M. Bykov et al., Angew. Chem. Int. Ed. 55, 15053 (2016).]. The latter was shown to include the first example of fivefold $\mathrm{O}$-coordinated $\mathrm{P}$ atoms in an inorganic phosphate compound. In this work, we characterize the electronic structure and other physical properties of these phases by means of $a b$ initio calculations and investigate the structural transition. We find that the appearance of phases IV and V coincides with a collapse of the Mott insulating gap and quenching of magnetism in phase III as pressure is applied. Remarkably, our calculations show that in the high-pressure phase $\mathrm{V}$, these features reappear, leading to an antiferromagnetic Mott insulating phase, with robust local moments.
\end{abstract}

DOI: 10.1103/PhysRevB.99.245132

\section{INTRODUCTION}

Modern advances in experimental high-pressure techniques have lead to numerous discoveries of new phenomena and previously unknown phases of materials [1-3]. Recently, experiments based on synchrotron x-ray diffraction in diamond anvil cells [4] found two high-pressure phases of $\mathrm{TiPO}_{4}$. One of these new phases, phase V, showed experimental confirmation of fivefold $\mathrm{O}$-coordinated $\mathrm{P}$ atoms in an inorganic phosphate compound.

At ambient conditions, $\mathrm{TiPO}_{4}$ crystallizes in the orthorhombic $\mathrm{CrVO}_{4}$ structure (space group $\mathrm{Cmcm}$ ), which features $\mathrm{TiO}_{6}$ and $\mathrm{PO}_{4}$ polyhedra [Fig. 1(a)] and is referred to as phase I [4]. The edge-sharing $\mathrm{TiO}_{6}$ octahedra form quasi-one-dimensional (1D) magnetic chains along the $c$ axis. By means of nuclear magnetic resonance measurements in combination with $a b$ initio calculations, magnetic coupling along these chains has been determined to be antiferromagnetic (AFM) [5]. $\mathrm{TiPO}_{4}$ has attracted attention because it is one of only a few inorganic materials undergoing a spin-Peierls (SP) transition with a particularly high critical temperature of $T_{S P}=74 \mathrm{~K}$ [5-7], which can be compared to $T_{S P}=14 \mathrm{~K}$ in $\mathrm{CuGeO}_{3}$ and $34 \mathrm{~K}$ in $\mathrm{NaV}_{2} \mathrm{O}_{5}$ [8,9]. The temperature of the $\mathrm{SP}$ transition in $\mathrm{TiPO}_{4}$ appears to be sensitive to the applied pressure. Ambient temperature compression to $4.5 \mathrm{GPa}$ leads to an incommensurately modulated structure (phase II) that is seen upon cooling below $111 \mathrm{~K}$ at ambient pressure. The commensurate spin-Peierls phase III [Fig. 1(b)] becomes stabilized at $7.3 \mathrm{GPa}$ [4] at room temperature.

Phase III can be regarded as a fourfold superstructure of phase $\mathrm{I}$ and features the same $\mathrm{TiO}_{6}$ and $\mathrm{PO}_{4}$ polyhedra. However, above $46 \mathrm{GPa}$, the diffraction pattern has been reported to change drastically, revealing a structural transition into two coexisting phases, called IV and V [Figs. 1(c) and 1(d)] [4]. Phase IV is similar to phase III but with a denser hexagonal packing of the $\mathrm{O}$ atoms. This means that the $\mathrm{TiO}_{6}$ octahedra become highly distorted, leading to $\mathrm{TiO}_{7}$ capped trigonal prisms while the $\mathrm{PO}_{4}$ tetrahedra are retained. Phase $\mathrm{V}$ has monoclinic $\mathrm{P} 2_{1} / m$ symmetry, with interlinked $\mathrm{TiO}_{7}$ and the unprecedented $\mathrm{PO}_{5}$ polyhedra.

Little is known about these high-pressure phases because of the difficulties associated with conducting in situ measurements in diamond-anvil cells. In this work, we investigate the electronic structure of the novel high-pressure phases of $\mathrm{TiPO}_{4}$. In particular, we focus on the relative stability of phases IV and V, as well as orbital and magnetic ordering, and demonstrate the exciting Mott physics that occurs in highly compressed $\mathrm{TiPO}_{4}$. Our experiments reveal that upon compression the band gap in phase III shrinks, as indicated by a reddening of the color of the samples. At the transition to phases IV and V, the sample contains a mix of phases IV and $\mathrm{V}$, and its color turns black. Ab initio calculations show that the gap is indeed closed in phases III and IV at high pressure. However, phase $\mathrm{V}$ features a sizable Mott gap and magnetic moments.

\section{EXPERIMENTAL DETAILS}

A single crystal of $\mathrm{TiPO}_{4}$ from the same synthetic batch that was used for the high-pressure studies in Ref. [4] was placed inside a sample chamber in a BX90 diamond anvil cell. Neon was used as a pressure-transmitting medium, while a small chip of ruby was used as a pressure standard [10]. The sample was compressed in a few steps up to $51 \mathrm{GPa}$ to track the color change of the crystal upon compression. 


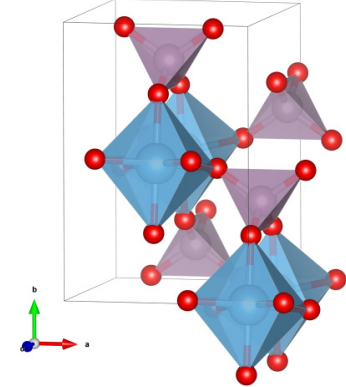

(a)

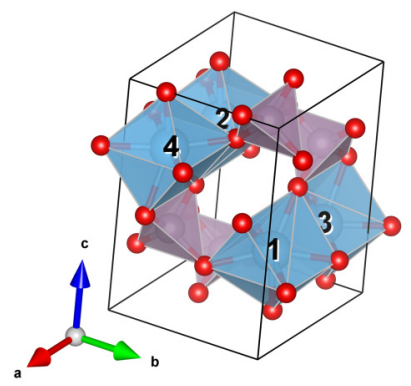

(c)

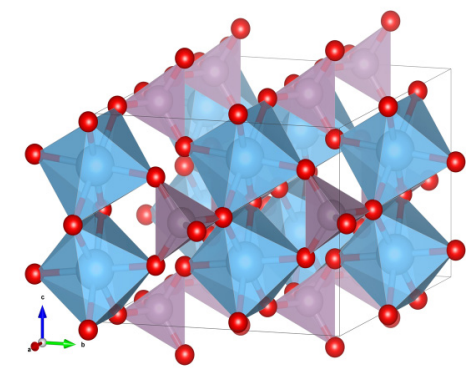

(b)

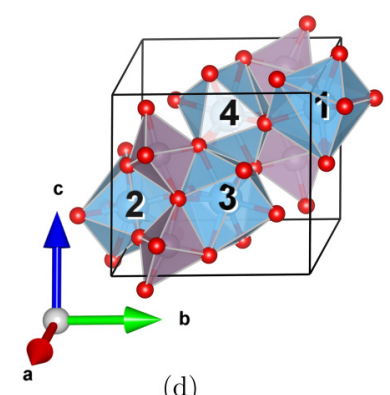

(d)
FIG. 1. Crystal structures observed in $\mathrm{TiPO}_{4}$ under increasing compression: (a) phase I, (b) phase III, (c) phase IV, and (d) phase V. $\mathrm{Ti}$ atoms are shown in blue, $\mathrm{P}$ are purple, and $\mathrm{O}$ are red. Also shown in blue are the Ti-O polyhedra and in purple are the $\mathrm{P}-\mathrm{O}$ polyhedra.

\section{COMPUTATIONAL DETAILS}

Calculations were performed using the projector augmented wave $[11,12]$ (PAW) method implementation in the Vienna $a b$ initio Simulations Package $[13,14]$ (VASP). For the antiferromagnetic calculations, we employed a plane wave energy cutoff of $520 \mathrm{eV}$. The calculations employed the local density approximation [15] (LDA) with an added onsite effective interaction parameter, $U(\mathrm{LDA}+U)$ as parametrized by Dudarev et al. [16]. Calculations of phase I employed a $k$ point mesh of $10 \times 8 \times 10 k$ points using the Monkhorst-Pack

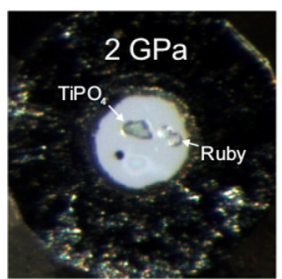

(a)

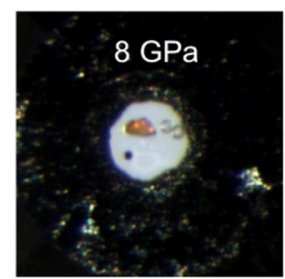

(b)

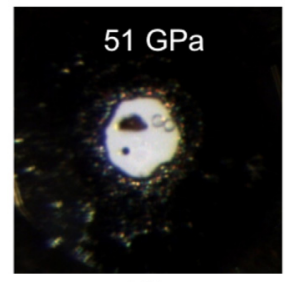

(d)

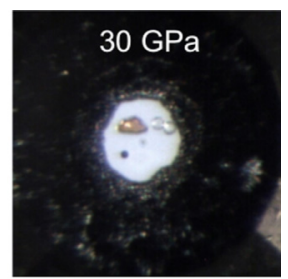

(c)
FIG. 2. Sample of $\mathrm{TiPO}_{4}$ at different pressures. Note the color change as pressure is increased.

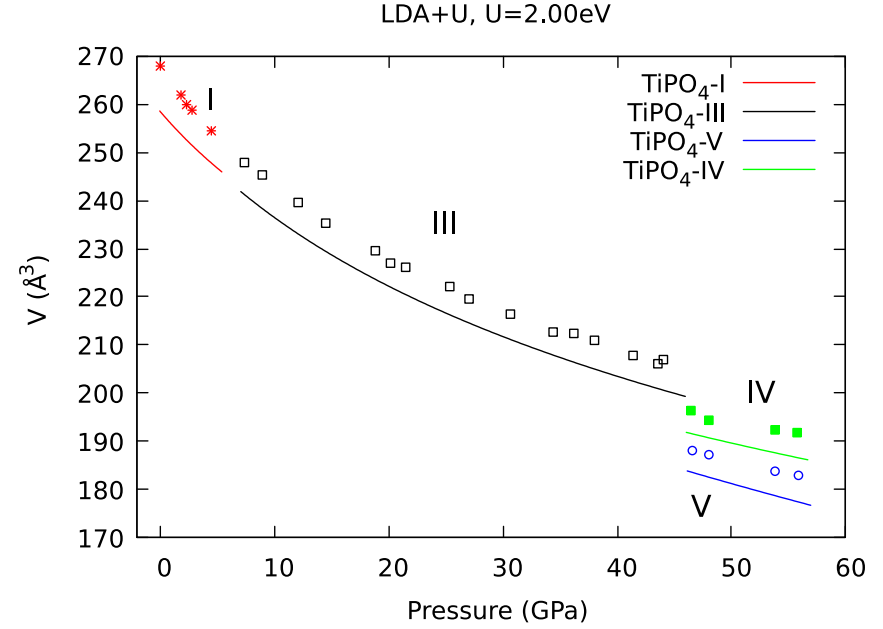

FIG. 3. Equation of state for $\mathrm{TiPO}_{4}$. Points are experimental data [4]. Solid lines show EOS calculated in this work.

scheme [17], with a total of $200 k$ points in the irreducible Brillouin zone. For phase III, we employed a $k$-point mesh of $5 \times 7 \times 11 k$ points, resulting in a total of $112 k$ points in the irreducible Brillouin zone. For the structural optimization of phases IV and $\mathrm{V}$, we employed $k$-point meshes of $12 \times$ $10 \times 8$ and $7 \times 4 \times 5$, resulting in 240 and $70 k$ points in the respective irreducible Brillouin zones. For the calculations of the electronic structure of phase $\mathrm{V}$ a refined $k$-point mesh of $9 \times 7 \times 5$, giving $158 k$-points in the irreducible Brillouin zone, was used.

Calculated energies and volumes were fitted to the thirdorder Birch-Murnaghan equation of state (EOS) [18]. We chose the value $U=2.0 \mathrm{eV}$ because it adequately reproduces the experimental equation of state, as demonstrated below, as well as the quite complex pressure dependence of the crystal structure parameters of phase III, as will be presented elsewhere.

\section{RESULTS}

Figure 2 shows the change in color of the $\mathrm{TiPO}_{4}$ crystal on compression in a diamond anvil cell. Upon phase transformation to phase III, the color of the crystal changes from green [Fig. 2(a)] to orange [Figs. 2(b) and 2(c)], while the transition to phases IV and $\mathrm{V}$ results in the sample turning black. Similar changes has been observed in related ternary compunds $[19,20]$.

TABLE I. Equilibrium parameters from calculations and experiment. Experimental values for phases I, II, and III were determined in Ref. [4] from a single fit.

\begin{tabular}{lccc}
\hline \hline & $\mathrm{V}_{0}\left(\AA^{3}\right)$ & $\mathrm{B}_{0}(\mathrm{GPa})$ & $\mathrm{B}_{0}^{\prime}$ \\
\hline Phase I & 258.56 & 92 & 6.5 \\
Phase III & 258.79 & 87 & 5.4 \\
Experiment[4] (phases I, II, and III) & 267.86 & 72 & 6.5 \\
Phase IV & 236.46 & 133.11 & 4.8 \\
Phase V & 225.95 & 179 & 2.4 \\
\hline \hline
\end{tabular}




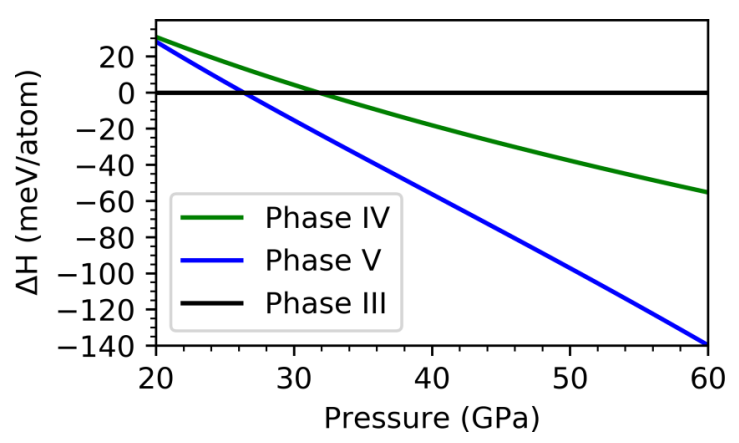

FIG. 4. Enthalpy for phases IV and V relative to phase III as a function of pressure.

In Fig. 3, we show the obtained volume-pressure relation for phases I, III, IV, and V. Phase I was set up in the same AFM configuration used in earlier calculations [21]. We considered phase III in the AFM configuration suggested by experiments [4]. For phases IV and V, we compare the calculated enthalpy, $H=E(V)+P V$, for four different magnetic configurations: ferromagnetic $\uparrow \uparrow \uparrow \uparrow$ as well as three antiferromagnetic configurations, $\downarrow \uparrow \uparrow \downarrow, \downarrow \uparrow \downarrow \uparrow$, and $\uparrow \uparrow \downarrow \downarrow$ (the order of Ti atoms is shown in Fig. 1). For phase IV, all configurations are within $2 \mathrm{meV} /$ atom of each other, with the AFM configuration $\downarrow \uparrow \downarrow \uparrow$ having the lowest enthalpy. In phase $\mathrm{V}$, the difference in enthalpy is at most $4 \mathrm{meV} /$ atom, with the AFM configuration $\downarrow \uparrow \uparrow \downarrow$ being the lowest. The difference in enthalpy is very

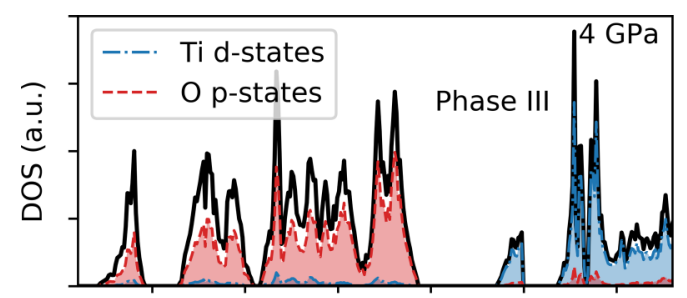

(a)

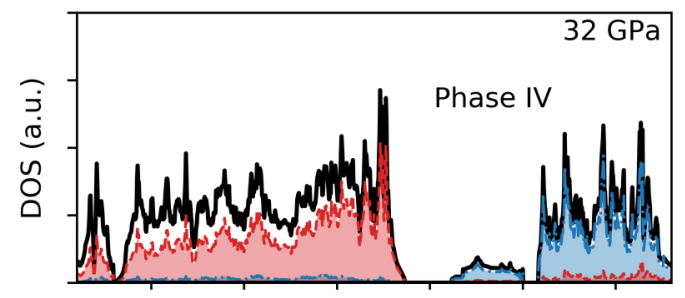

(c)

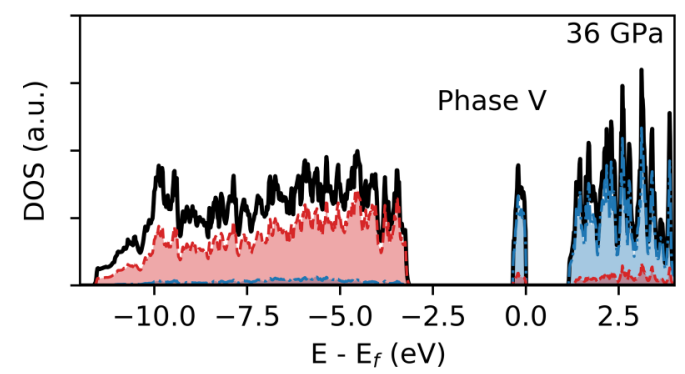

(e) small compared to typical thermal energy at room temperature $(k T \sim 20 \mathrm{meV})$. This means that at room temperature, phases IV and V are most likely in a paramagnetic state with disordered local moments.

There is a pressure-independent underestimation of the theoretical unit cell volume of about $4 \%$ compared to experiments. However, the overall behaviour of the EOS is very well reproduced. Our results for $B_{0}$ agree well with previous results obtained for phase I using the generalized gradient functional described by Perdew et al. [22], without on-site interaction $U$ [21]; however, we obtain a lower value of $V_{0}$. This should be seen in the light that our calculations do not include thermal effects and that the PBE functional fails to reproduce an insulating ground state for phase III. The transition from phase III to phases IV and $\mathrm{V}$ includes a decrease in the unit cell volume. In Table I, we summarize the equilibrium parameters obtained from the EOS fits for the different phases. Note that for phase IV and $\mathrm{V}$ there is a decrease in equilibrium volume and a significant increase in bulk modulus. Comparing phases III and V, there is a twofold increase of $B_{0}$ for the high-pressure phase. In Fig. 4, we show the enthalpies of phases IV and V relative to phase III. We see that phase V becomes favorable over phase III at around $25 \mathrm{GPa}$, and phase IV becomes favorable over phase III at around $33 \mathrm{GPa}$. In (room-temperature) experiments, these two phases have been reported to appear at around $45 \mathrm{GPa}$. The underestimation of the transition pressure is expected because of the systematic underestimation of the volume in the EOS (the pressure is thus

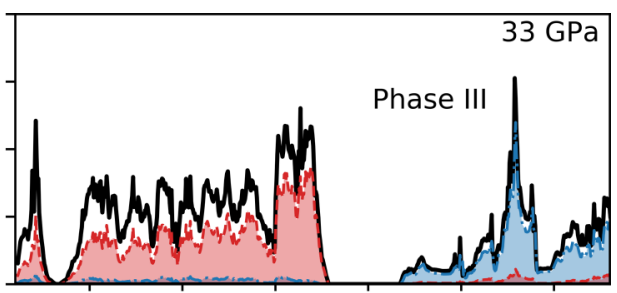

(b)

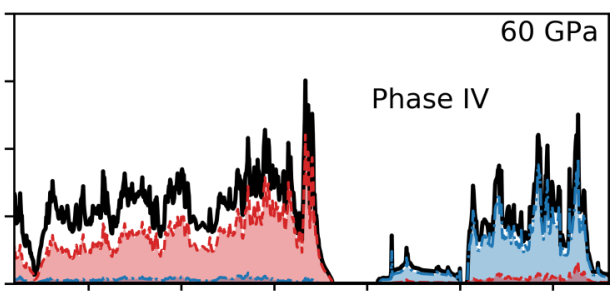

(d)

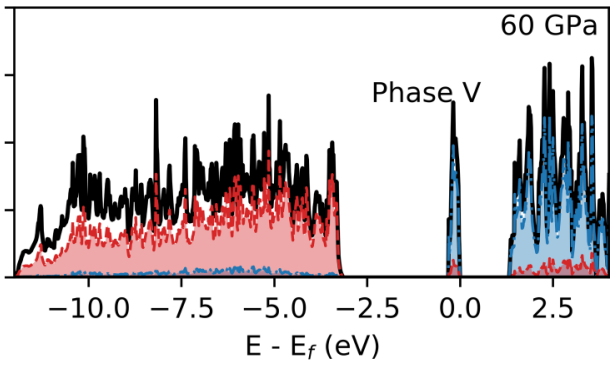

(f)

FIG. 5. Black line refers to the total DOS of the unit cell. Blue and red refer to the site and orbital-projected DOS. Energy given relative to the Fermi energy $\left(E_{F}\right)$. 


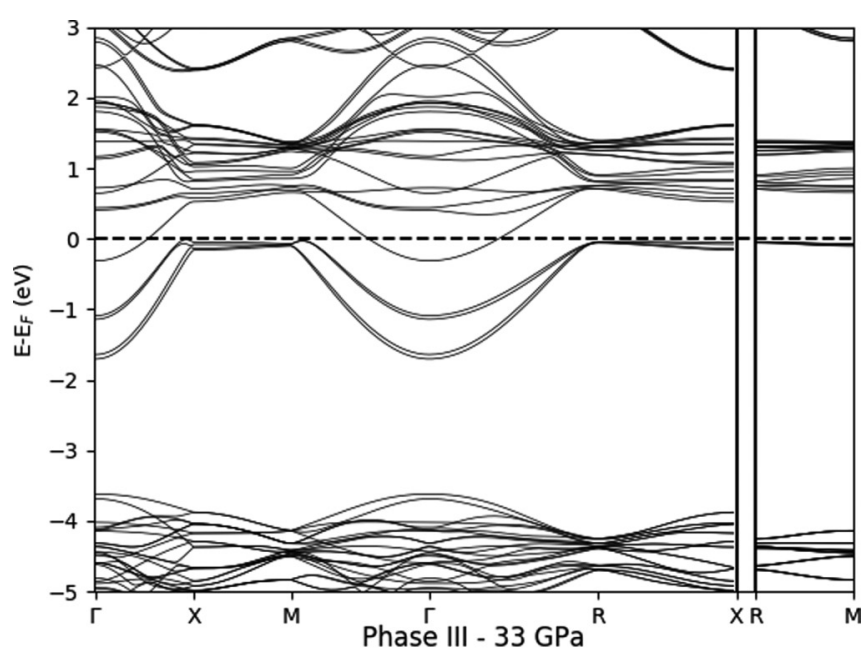

(a)

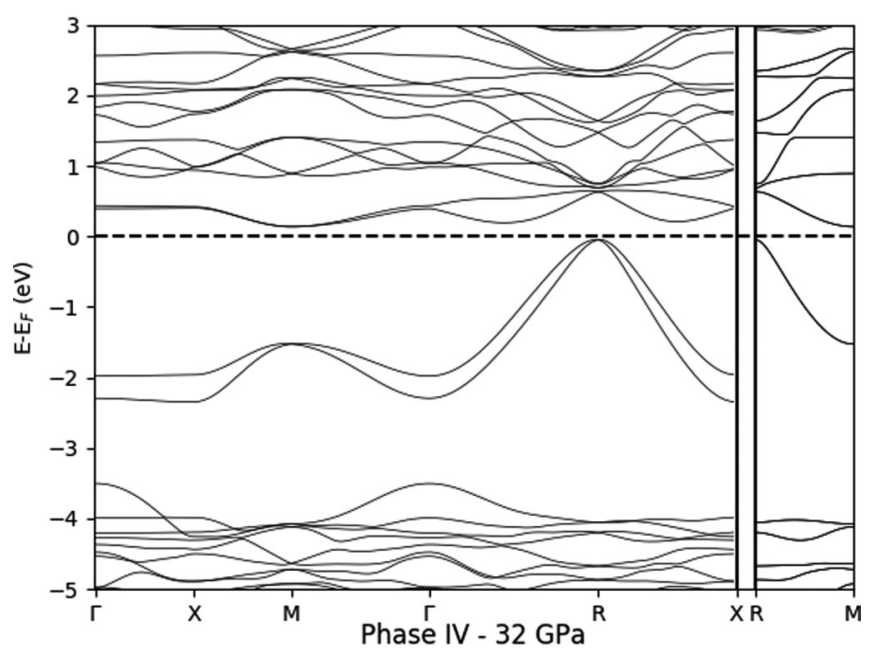

(b)

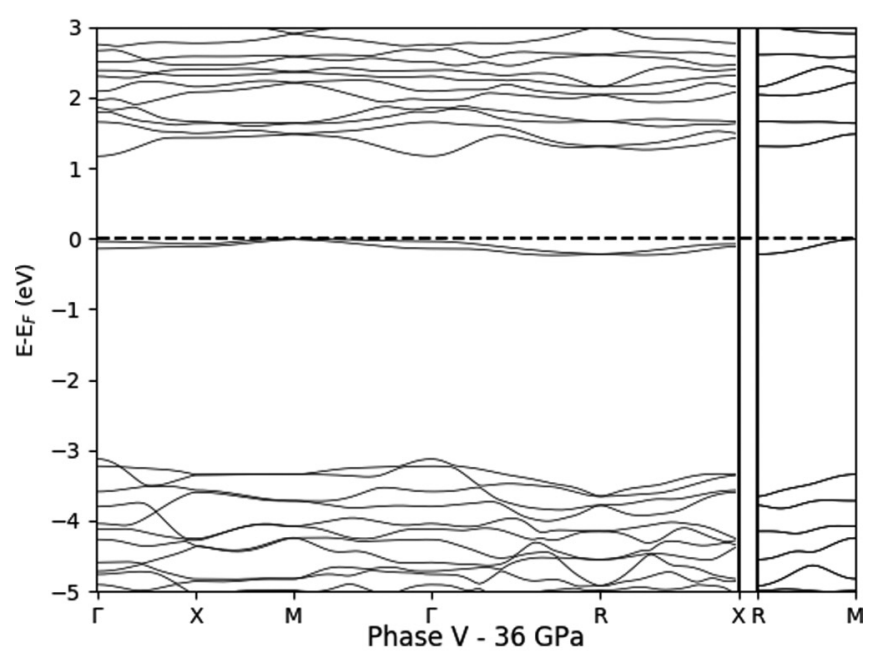

(c)

FIG. 6. Calculated band stucture for $\mathrm{TiPO}_{4}$ phases (a) III at $33 \mathrm{GPa}$, (b) IV at $32 \mathrm{GPa}$, and (c) V at $36 \mathrm{GPa}$. The Fermi energy is set to the top of the valence band.

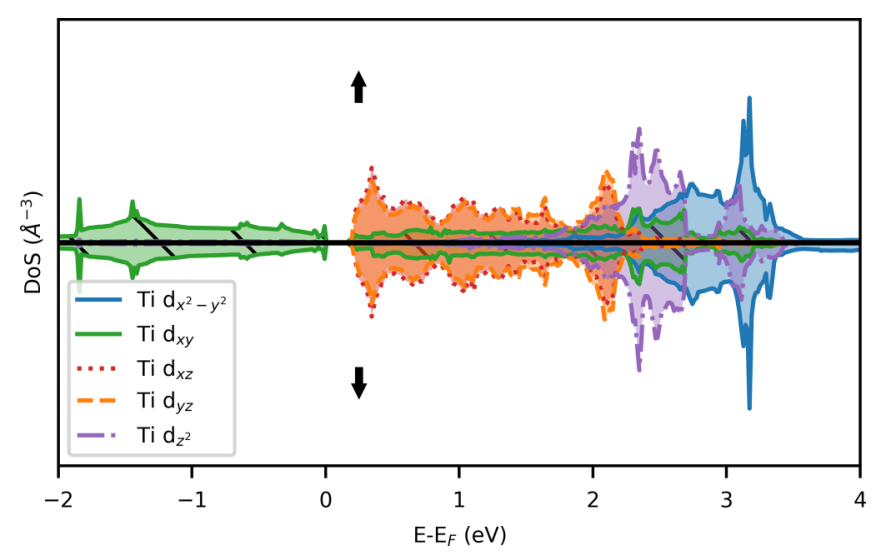

FIG. 7. Calculated spin-resolved total DOS for one Ti atom in phase IV, at $60 \mathrm{GPa}$.

too low at each volume). As outlined in Ref. [4], the transition from phase III to phase IV involves only small changes to the crystal structure, whereas the transition from phase III to $\mathrm{V}$ involves substantial rearrangement. Interestingly, phase $\mathrm{V}$ is lower in enthalpy than phase IV in the entire pressure range. Thus, it appears that phase $\mathrm{V}$ is the thermodynamically stable phase at high pressures, while phase IV is kinetically stable, which explains the coexistence of the two structures in the high-pressure experiment [4]. Phase IV maintains the alternating Ti-Ti distances along the $a$ direction observed in the SP phase III [4].

Phase III is stable for a large range of pressures. Figure 5(a) shows its calculated density of states (DOS) at $4 \mathrm{GPa}$. A band gap between the occupied and unoccupied states is clearly visible. This is in line with experimental observations (Fig. 2) that the samples are transparent at this pressure. As pressure is increased, the gap at the Fermi level shrinks, also in agreement with experiment. In Fig. 5(b), we see that at a pressure around $33 \mathrm{GPa}$ the gap in phase III is completely closed. This pressure is lower than the experimental pressure at which samples lose their transparency. However, it is the same pressure at which phases IV and V become energetically favorable in calculations. Although calculations underestimate the transition pressure, we conclude that, in agreement with experiment, the closing of the gap and the structural transition from phase III to phases IV and V coincide.

Figure 6 shows the calculated band structure for phases III, IV, and V. We observe that in all phases the band gap is indirect and that phases III and IV shows wider bands just below the Fermi energy than phase V.

In Fig. 5(c), we show the calculated DOS for $\mathrm{TiPO}_{4}$ phase $\mathrm{IV}$ at a pressure of $32 \mathrm{GPa}$. A very small band gap of $0.4 \mathrm{eV}$ is visible. The low-binding energy part of the spectrum is of mainly Ti- $d$ character, corresponding to a singly occupied $d_{x y}$ orbital, as shown in Fig. 7. This is separated from a high-binding part of filled O-p states. In Fig. 8, we show the occupied $d$ orbitals plotted in real space. There is a clear overlap of bonding orbitals in Ti chains along the $a$ direction, indicating direct exchange coupling. Note the separation of charge between Ti pairs, due to the alternating Ti-Ti distances. In Fig. 5(d), we show the calculated DOS of phase IV at a pressure of $60 \mathrm{GPa}$. The band gap has been reduced to 


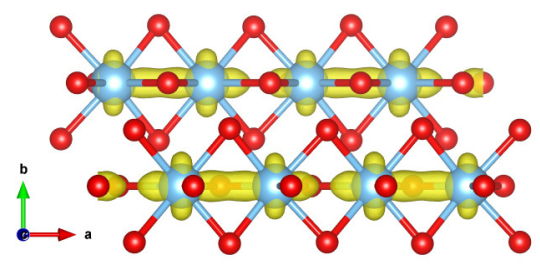

FIG. 8. Partial charge density of Ti- $d$ electrons in phase IV, at $60 \mathrm{GPa}$. The local $\bar{x}, \bar{y}$, and $\bar{z}$ axes have been chosen so that the $\bar{z}$ and $\bar{x}$ axes point toward oxygen atoms.

$0.15 \mathrm{eV}$. Indeed, the samples are seen in experiments to be dark [Fig. 2(d)].

In Fig. 5(e), the calculated DOS for phase $\mathrm{V}$ at a pressure of $36 \mathrm{GPa}$ is shown. The DOS for phase $\mathrm{V}$ at $58 \mathrm{GPa}$ is shown Fig. 5(f). A very narrow, singly occupied, $d$-level is separated from a high binding energy manifold. A wide insulating gap separates the occupied and unoccupied $d$ orbitals. Experimentally the samples seem to be darker, almost black. It should be noted that the sample contains a mixture of phases IV and $\mathrm{V}$ at this point, and our calculations indicate that the gap in phase IV should be very small, making phase IV appear black. Phase V has in contrast a larger gap and should thus be transparent. In the sample, the black phase IV is likely obscuring the transparent phase $\mathrm{V}$.

The local DOS for one Ti atom in phase $\mathrm{V}$ is shown in Fig. 9. Because of the pentagonal bipyramidal oxygen complex surrounding the Ti atom, the degeneracy of the different $d$ orbitals is lifted. The occupied $d$ orbitals are seen to be of $d_{x z}$ character, which is separated from the the unoccupied $d_{y z}$ orbital, pointing between $\mathrm{O}$ atoms. It should be noted that the local $d_{x z}$ orbitals of Ti nearest neighbors do not overlap to the same extent as in phase IV.

The gap between occupied and unoccupied $d$ states appear only if a nonzero on-site $U$ is included in the calculation. The separation of $d$ orbitals into upper and lower Hubbard bands is thus due to strong correlation between $d$ electrons. The filled $\mathrm{O}$ states are well below the low-binding energy $d$ states. Therefore, phase V may be classified as a true Mott insulator [23].

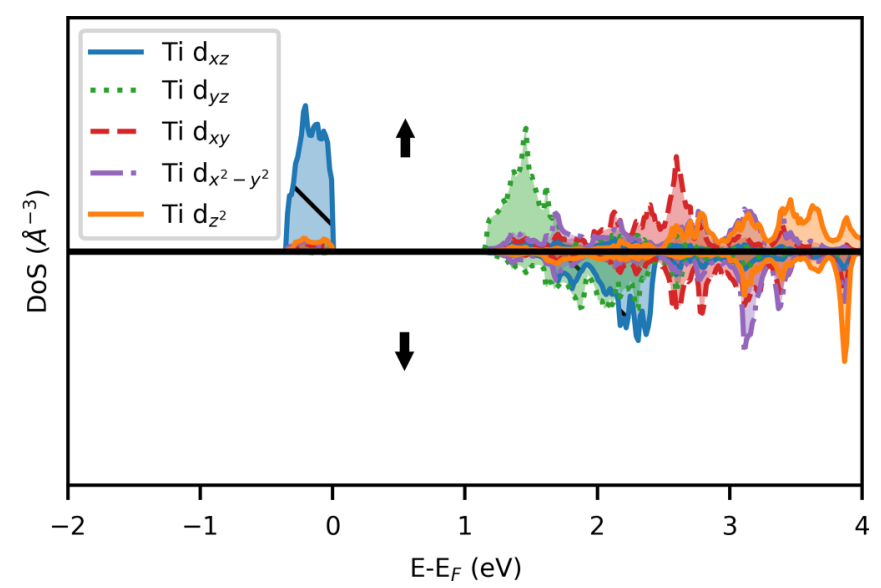

FIG. 9. Calculated spin-resolved DOS for one Ti atom in phase $\mathrm{V}$, at $36 \mathrm{GPa}$.

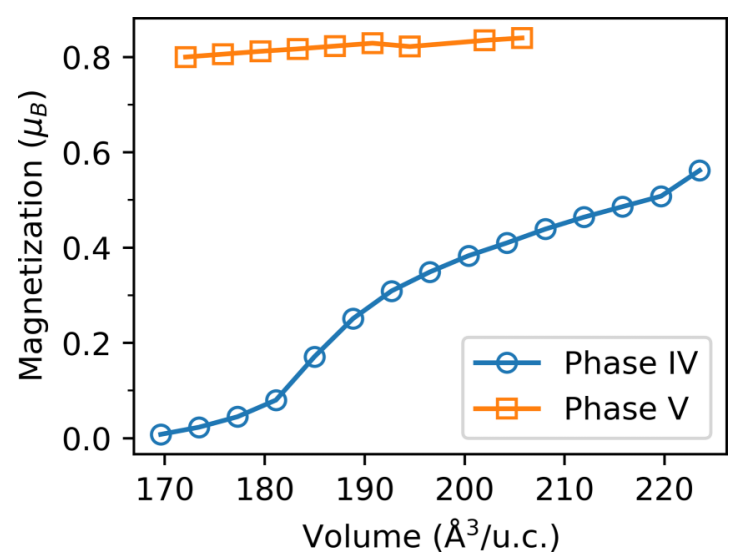

FIG. 10. Calculated local magnetic moments as a function of unit cell volume. For phases IV and V in AFM configuration.

The magnitude of the gap will depend strongly on the choice of exchange-correlation functional [24-26] and in this case also on the choice of the $U$ parameter. Nevertheless, $U=2.0 \mathrm{eV}$ seems to adequately reproduce the EOS and simultaneously reproduce the insulating state of phase III (and I). The gap is intact at the volumes where these phases were observed. Thus, the qualitative result of a nonmetallic state reappearing in the high-pressure phases is not sensitive to the specific value of $U$.

The results strongly suggests that the structural transition is connected with the Mott transition. The transformation into a metallic state destabilizes phase III, leading to phases IV and $\mathrm{V}$, which do have Mott gaps.

Early experimental studies [27] of the magnetic properties of $\mathrm{TiPO}_{4}$ reported a local magnetic moment on $\mathrm{Ti}^{3+}$ of $0.8 \mu_{B}$ at ambient pressure. This is only slightly lower than what is expected from an insulator with $\mathrm{Ti} d^{1}$ configuration. By means of ab initio calculations, Lopez et al. [21] reported the magnetic moment to vanish at $12 \mathrm{GPa}$ in the $\mathrm{CrVO}_{4}$ structure (phase I). Our calculations indicate that close to the collapse of phase III, the local magnetic moment is effectively zero.

The calculated magnetic moments for phases IV and V are shown in Fig. 10 as a function of unit cell volume. At the

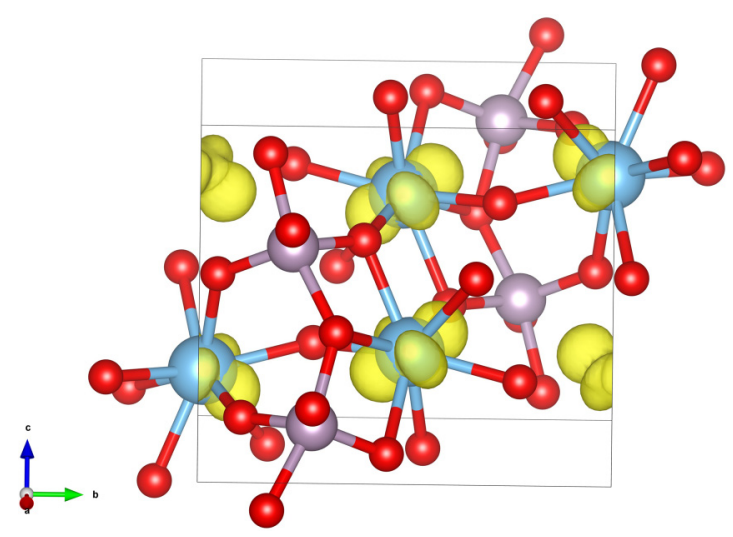

FIG. 11. Partial charge density of Ti- $d$ electrons in phase V, at $60 \mathrm{GPa}$. The local $\bar{x}, \bar{y}$, and $\bar{z}$ axes have been chosen so that the $\bar{z}$ and $\bar{x}$ axes point toward oxygen atoms. 
volume where phase IV becomes stable, the local magnetic moments are small, $0.3 \mu_{B}$, and continuously decrease with pressure. At $65 \mathrm{GPa}$, they have practically vanished, which is also indicated by the depolarized $d$-level DOS in Fig. 7. On the contrary, in phase $\mathrm{V}$ the magnetic moments are comparatively large, $0.8 \mu_{B}$, and decrease very slowly with increasing pressure. Because of the crystallographic geometry, there is no overlap between neighboring $d_{x z}$ states (Fig. 11). The AFM order therefore seems to be due to indirect Ti-O-Ti exchange coupling. Phase V is thus clearly antiferromagnetic, and the Ti atoms feature a robust local magnetic moment, compatible with the $d^{1}$ configuration.

\section{CONCLUSIONS}

Calculations show that the recently discovered phase $\mathrm{V}$ of $\mathrm{TiPO}_{4}$, featuring $\mathrm{PO}_{5}$ polyhedra, is the energetically favorable structure at high pressure, as compared to phases III and IV. The less dense phase IV is most likely kinetically stabilized. These phases appear when the pressure approaches values at which the known phase III should become metallic and lose its local magnetic moment. Phase IV shows a larger band gap, but otherwise behaves similarly to phase III. In contrast, phase $\mathrm{V}$ shows a band gap, and in phase $\mathrm{V}$ magnetism reappears. The calculations also show that phases III, IV, and V all have indirect band gaps. These results agree well with the color changes observed in experiments, though the small gap of phase IV obscures the transparent phase $\mathrm{V}$ in the experimental sample containing a mixture of the two phases. At present, we cannot justify a direct correspondence between the observed color and the size of the fundamental band gap. In general, there may be a difference between the optical and fundamental gap [28]; in the present case, this would require further studies of optical properties of $\mathrm{TiPO}_{4}$. Therefore, we refrain from direct comparisons of observed color and the size of the fundamental gap.

Our finding is in many ways remarkable, as pressureinduced structural transitions in Mott insulators are usually connected with the disappearance of the band gap. In phase $\mathrm{V}$ of $\mathrm{TiPO}_{4}$, the gap along with magnetism reappear upon a structural transition. The density of phase $\mathrm{V}$ is also higher than that of phases III or IV and our calculations show that phase $\mathrm{V}$ is the stable phase at high pressures. Thus, it should be possible to detect the inverse pressure-induced Mott transition in $\mathrm{TiPO}_{4}$ experimentally. In summary, our study shows that $\mathrm{TiPO}_{4}$ displays intriguing phenomena not only at low temperature but also at high pressure.

\section{ACKNOWLEDGMENTS}

This project is funded by the Knut and Alice Wallenberg Foundation for the project Strong Field Physics and New States of Matter (Grant No. KAW-2013.0020). We are grateful to the Swedish e-Science Research Centre (SeRC) for financial support. I.A.A. gratefully acknowledges the Swedish Research Council (VR) Grant No. 2015-04391 and the Swedish Government Strategic Research Area in Materials Science on Functional Materials at Linköping University (Faculty Grant SFO-Mat-LiU No. 2009 00971). Theoretical analysis of computational results was supported by the Russian Science Foundation (Project No. 18-12-00492). The computations were performed on resources provided by the Swedish National Infrastructure for Computing (SNIC) at High Performance Computing Center North (HPC2N) and National Supercomputer Centre (NSC).
[1] L. Dubrovinsky, N. Dubrovinskaia, E. Bykova, M. Bykov, V. Prakapenka, C. Prescher, K. Glazyrin, H. P. Liermann, M. Hanfland, M. Ekholm, Q. Feng, L. V. Pourovskii, M. I. Katsnelson, J. M. Wills, and I. Abrikosov, Nature (London) 525, 226 (2015).

[2] M. Bykov, E. Bykova, G. Aprilis, K. Glazyrin, E. Koemets, I. Chuvashova, I. Kupenko, C. McCammon, M. Mezouar, V. Prakapenka, H.-P. Liermann, F. Tasnadi, A. V. Ponomareva, I. Abrikosov, N. Dubrovinskaia, and L. Dubrovinsky, Nat. Commun. 9, 2756 (2018).

[3] I. Troyan, A. Gavriliuk, R. Ruffer, A. Chumakov, A. Mironovich, I. Lyubutin, D. Perekalin, A. P. Drozdov, and M. I. Eremets, Science 351, 1303 (2016).

[4] M. Bykov, E. Bykova, M. Hanfland, H.-P. Liermann, R. K. Kremer, R. Glaum, L. Dubrovinsky, and S. van Smaalen, Angew. Chem. Int. Ed. 55, 15053 (2016).

[5] J. M. Law, C. Hoch, R. Glaum, I. Heinmaa, R. Stern, J. Kang, C. Lee, M.-H. Whangbo, and R. K. Kremer, Phys. Rev. B 83, 180414(R) (2011).

[6] D. Wulferding, A. Möller, K.-Y. Choi, Y. G. Pashkevich, R. Y. Babkin, K. V. Lamonova, P. Lemmens, J. M. Law, R. K. Kremer, and R. Glaum, Phys. Rev. B 88, 205136 (2013).

[7] M. Bykov, J. Zhang, A. Schönleber, A. Wölfel, S. I. Ali, S. van Smaalen, R. Glaum, H.-J. Koo, M.-H. Whangbo, P. G.
Reuvekamp, J. M. Law, C. Hoch, and R. K. Kremer, Phys. Rev. B 88, 184420 (2013).

[8] M. Hase, I. Terasaki, and K. Uchinokura, Phys. Rev. Lett. 70, 3651 (1993).

[9] M. Isobe and Y. Ueda, J. Phys. Soc. Jpn. 65, 1178 (1996).

[10] H. K. Mao, J. Xu, and P. M. Bell, JGR: Solid Earth 91, 4673 (1986).

[11] P. E. Blöchl, Phys. Rev. B 50, 17953 (1994).

[12] G. Kresse and D. Joubert, Phys. Rev. B 59, 1758 (1999).

[13] G. Kresse and J. Furthmüller, Phys. Rev. B 54, 11169 (1996).

[14] G. Kresse and J. Furthmüller, Comput. Mater. Sci. 6, 15 (1996).

[15] J. P. Perdew and A. Zunger, Phys. Rev. B 23, 5048 (1981).

[16] S. L. Dudarev, G. A. Botton, S. Y. Savrasov, C. J. Humphreys, and A. P. Sutton, Phys. Rev. B 57, 1505 (1998).

[17] H. J. Monkhorst and J. D. Pack, Phys. Rev. B 13, 5188 (1976).

[18] F. Birch, Phys. Rev. 71, 809 (1947).

[19] D. Errandonea, E. Bandiello, A. Segura, J. J. Hamlin, M. B. Maple, P. Rodriguez-Hernandez, and A. Muñoz, J. Alloys Compd. 587, 14 (2014).

[20] M. A. Hakeem, D. E. Jackson, J. J. Hamlin, D. Errandonea, J. E. Proctor, and M. Bettinelli, Inorg. Chem. 57, 7550 (2018).

[21] S. López-Moreno and D. Errandonea, Phys. Rev. B 86, 104112 (2012). 
[22] J. P. Perdew, K. Burke, and M. Ernzerhof, Phys. Rev. Lett. 77, 3865 (1996).

[23] J. Zaanen, G. A. Sawatzky, and J. W. Allen, Phys. Rev. Lett. 55, 418 (1985).

[24] A. Benmakhlouf, D. Errandonea, M. Bouchenafa, S. Maabed, A. Bouhemadou, and A. Bentabet, Dalton Trans. 46, 5058 (2017).

[25] R. Lacomba-Perales, D. Errandonea, A. Segura, J. RuizFuertes, P. Rodríguez-Hernández, S. Radescu, J. López-Solano,
A. Mujica, and A. Muñoz, J. Appl. Phys. 110, 043703 (2011).

[26] J. P. Perdew, Int. J. Quantum Chem. 28, 497 (1985).

[27] N. Kinomura, F. Muto, and M. Koizumi, J. Solid State Chem. 45, 252 (1982).

[28] A. Walsh, J. L. F. Da Silva, S.-H. Wei, C. Körber, A. Klein, L. F. J. Piper, A. DeMasi, K. E. Smith, G. Panaccione, P. Torelli, D. J. Payne, A. Bourlange, and R. G. Egdell, Phys. Rev. Lett. 100, 167402 (2008). 